Research Paper

\title{
Chemokine Receptors CXCR4 and CXCR7 are Associated with Tumor Aggressiveness and Prognosis in Extramammary Paget Disease
}

\author{
Kun Chang1*, Gao-Xiang Li1*, Yun-Yi Kong2*, Xu-Xia Shen ${ }^{2}$, Yuan-Yuan Qu1 ${ }^{1}$, Zhong-Wei Jia1, Yue Wang1, \\ Bo Dai ${ }^{1 凶}$, Ding-Wei Ye ${ }^{1 凶}$ \\ 1. Department of Urology, Fudan University Shanghai Cancer Center; Department of Oncology, Shanghai Medical College, Fudan University, Shanghai \\ 200032, China; \\ 2. Department of Pathology, Fudan University Shanghai Cancer Center; Department of Oncology, Shanghai Medical College, Fudan University, Shanghai \\ 200032, China. \\ * Equal contributors \\ $\square$ Corresponding authors: Bo Dai, Department of Urology, Fudan University Shanghai Cancer Center, 270 Dong'An Road, Shanghai 200032, China. E-mail: \\ bodai1978@126.com Ding-Wei Ye, Department of Urology, Fudan University Shanghai Cancer Center, 270 Dong'An Road, Shanghai 200032, China. E-mail: \\ dwyeli@163.com \\ (c) Ivyspring International Publisher. This is an open access article distributed under the terms of the Creative Commons Attribution (CC BY-NC) license \\ (https:// creativecommons.org/licenses/by-nc/4.0/). See http://ivyspring.com/terms for full terms and conditions.
}

Received: 2017.01.10; Accepted: 2017.05.10; Published: 2017.08.02

\begin{abstract}
Chemokines are involved in many aspects of oncogenesis, including regulation of cancer cell growth, dissemination and host-tumor response. However, the potential of the chemokine receptors, CXCR4 and CXCR7, in serving as biomarkers in extramammary Paget's disease (EMPD) has been rarely examined. Expressions of CXCR4 and CXCR7 were evaluated in 92 EMPD specimens by immunohistochemistry. High expression of CXCR4 and CXCR7 were both correlated with regional lymph node metastasis and presence of lymphovascular invasion. High expression of CXCR7 also correlated with the depth of invasion. The prognostic value of these two chemokines were also investigated in progression-free survival (PFS) and cancer-specific survival (CSS). Both high expression of CXCR4 and CXCR7 were indicative of shorter PFS and CSS. In the combined prognostic model, concomitant high expression of CXCR4 and CXCR7 were suggestive of poor prognosis compared with the other two groups. In the multivariate analysis, depth of invasion, combined prognostic model and regional lymph node metastasis at diagnosis were the independent prognostic factors for EMPD patients for PFS, and the former two factors independently impacted CSS. Our results demonstrated that CXCR4 and CXCR7 can be used as prognostic biomarkers and prediction of aggressiveness of EMPD. Therapy targeting CXCR4 and CXCR7 may helpful to prevent EMPD progression and improve the prognosis of EMPD.
\end{abstract}

Key words: EMPD, CXCR7, CXCR4, invasive, prognosis.

\section{Introduction}

Extramammary Paget's disease (EMPD) is a rare cutaneous malignant neoplasm of unknown histogenesis. It mostly arises in areas rich in apocrine sweat glands, e.g. the anogenital areas, the perineal area and axilla. ${ }^{[1,2]}$ Early lesions generally present as red or brown plaques and the lesions become erosive, infiltrated in the late stage. Patients classified as regional intraepithelial invasion experience good prognosis regardless of tumor diameter. ${ }^{[4-6]}$ However, in some instances, EMPD tumor cells invade deeply into the dermis and develop nodules and ulcerations. Tumors then rapidly metastasize to regional lymph nodes and distant organs, leading to poor outcomes. ${ }^{[4-7]}$ Treatment for advanced EMPD with systemic metastasis often ends up with a disappointing result, as no highly effective therapy has been established. Identification of reliable prognostic molecular markers for EMPD could 
provide novel therapeutic targets, which are critically important for developing molecular profile-directed therapies for patients with EMPD.

Chemokines belong to a superfamily of chemo-attracting proteins that bind to chemokine receptors. They are involved in many aspects of oncogenesis, including regulation of cancer cell growth, dissemination and host-tumor response. ${ }^{[8,9]}$ Alterations of chemokine expression levels are significantly associated with more aggressive disease and worse prognosis in diverse malignancies. ${ }^{[10,11]}$ CXCR7 and CXCR4 shared the same chemokine ligands, CXCL12,12] with demonstrated critical roles in tumor growth, angiogenesis, and tumor cell homing in lymph nodes and distant metastases in a variety of solid human malignancies, such as lung cancer, ${ }^{[13]}$ breast cancer, ${ }^{[13]}$ prostate cancer, ${ }^{[14]}$ rhabdomyosarcomas ${ }^{[15]}$ and human glioma, ${ }^{16]}$ among others. In different tumor cell types, depending on the differentiation status and environment, they may function uniquely or even in combination. Based on these findings, we speculated whether CXCR4 and CXCR7 participate in the progression of EMPD and could serve as potential prognostic markers for the disease.

In this study, we investigated the expression of intratumoral CXCR4 and CXCR7 in a large cohort of EMPD patients. We evaluated the correlation between expression of these proteins and patient clinicopathological characteristics and assessed the prognostic value of these factors in progression-free survival (PFS) and cancer-specific survival (CSS).

\section{Materials and Methods}

\section{Patients and methods}

Patients with EMPD who underwent surgical excision of primary skin lesions at our institution between June 2003 and March 2013 were screened. All patients underwent a thorough physical examination and diagnostic tests, including cystoscopy, colonoscopy, chest X-ray, abdominal and pelvic CT before surgery. Patients who presented with synchronous or metachronous internal noncutaneous malignancies were excluded. In total, 92 patients who met these criteria and with available specimens were included in this study. Patients' clinical characteristics, such as age, sex, time delayed in diagnosis, lesion location, primary treatment, and adjuvant therapy, were recorded. Histopathological parameters, including longest diameter of lesion, surgical margin status, depth of invasion and lymphovascular invasion, were examined by two experienced pathologists. We stratified the patients into three groups according to depth of invasion: (i) intraepitheliar; (ii) upper dermis; and (iii) lower dermis. The pathological stage was determined according to the 7th edition of the American Joint Committee on Cancer TNM classification of skin appendageal carcinoma. ${ }^{[17]}$ Both inguinal and pelvic lymph nodes were deemed as regional nodes for the specific location of the lesion. PFS and CSS were assessed to determine the time to disease recurrence and cancer-related death, respectively. The end point for PFS was the date of local or regional recurrence or the date of distant metastasis. The end point for CSS was the date of cancer-related death. For CSS, patients who died of a cause unrelated to EMPD were considered as censored observations at the date of death. Hematoxylin and eosin sections were reviewed by pathologists to select representative areas of the original samples. The study was carried out in accordance with the ethical standards of the Helsinki Declaration II and approved by the Institution Review Board of Fudan University Shanghai Cancer Center. Informed consent was obtained from each patient and the study was approved by our Institutional Ethics Committee.

\section{Immunohistochemistry staining}

Immunostainings of CXCR4 and CXCR7 were performed using antibodies (CXCR4, Abcam, Cambridge, MA; dilution 1:50; CXCR7, Abgent, R\&D Systems, Abingdon, UK; dilution 1:200) and the Envision detection kit (Dako, Carpinteria, CA, USA). EMPD tissue sections $(4 \mu \mathrm{m})$ from archived formalin-fixed, paraffin-embedded tissue blocks were deparaffinized in a series of xylene and rehydrated in graded ethanol solutions. Endogenous peroxidase was quenched by incubation in $0.3 \% \mathrm{H}_{2} \mathrm{O}_{2}$ for $15 \mathrm{~min}$ at $37^{\circ} \mathrm{C}$ and nonspecific binding was blocked with $10 \%$ normal goat serum for $60 \mathrm{~min}$ at room temperature. For antigen retrieval, sections in $0.01 \mathrm{M}$ sodium citrate buffer, $\mathrm{pH}$ 6.0, were heated in a pressure cooker (20 psi for $10 \mathrm{~min}$ ). Incubation with primary antibodies was conducted overnight at $4^{\circ} \mathrm{C}$. Chromogenic detection was carried out using a peroxidase-conjugated secondary antibody (60 $\mathrm{min}$ ) and DAB reagents $(1 \mathrm{~min})$ provided in the Envision detection kit. Tissue sections were then counterstained with Meyer's Hematoxylin (Thermo Fisher Scientific, Waltham, MA, USA). The positive controls for CXCR7, and CXCR4 were conducted by using normal human tonsil and spleen tissues. The negative control was prepared using phosphate-buffered saline with omission of the primary antibody.

All slides were examined and scored by two experienced pathologists, who were blinded to the outcome and clinical data, in an open discussion. The 
CXCR4 and CXCR7 immunostaining was measured based on the intensity of staining (intensity score) and the quantity of immunoreactive cells (quantity score), as previously reported.[18] The intensity of immune staining was scored as 0 , negative; 1 , weak; 2, moderate; and 3, intense. The quantity of immunoreactive cells was scored as $0 \%$, none; 1 , $1 \%-30 \% ; 2,31 \%-60 \% ; 3,>60 \% .{ }^{[19]}$ The product of intensity score and quantity score was used as the total score, in which 0-3 indicates low expression, and 4-9 indicates high expression. ${ }^{20]}$

\section{Statistical analysis}

To evaluate the relationship between the immunostaining and clinicopathological parameters, the Chi-square test and Fisher's exact test were used. PFS and CSS were calculated by the Kaplan-Meier method with the log-rank test to assess differences between groups based on the gene expression status. Univariate and multivariate analysis of prognostic factors was performed using the Cox proportional hazard model. All statistical tests were performed using SPSS, version 20 (SPSS Inc., Chicago, IL). P < 0.05 was considered to indicate statistical significance.

\section{Results}

\section{Patient characteristics}

Among the total 92 patients recruited in the study, 87 were males and 5 were females. After a median (range) follow-up of 34 months (range 6-130 months), $25.0 \%(23 / 92)$ of the patients experienced clinical recurrence and 15.2\% (14/92) patients died of EMPD. Non-regional lymph nodes $(10.9 \%, 10 / 92)$ and bone $(7.6 \%, 7 / 92)$ were the most common sites of distant metastasis. The median PFS was 28 months (range 3 116 months) and the median CSS was 30 months (range 5 130 months). The detailed clinicopathological characteristics of the patients are summarized in Table 1.

\section{CXCR4 and CXCR7 expression in EMPD}

Immunohistochemical staining showed that both CXCR4 and CXCR7 were expressed in the cytoplasm in EMPD specimens. Among the 92 EMPD patients, $22(23.9 \%)$ and $21(22.9 \%)$ had high expression of CXCR4 and CXCR7, respectively. Representative examples of high and low CXCR4 and CXCR7 EMPD specimens are shown in Figure 1.

\section{Association of CXCR4 and CXCR7 expression with patient characteristics}

The clinicopathological characteristics of patients grouped by CXCR4 and CXCR7 expression level are listed in Table 1. High expression of CXCR4 was correlated with lymphovascular invasion $(\mathrm{P}=0.007)$ and regional lymph node metastasis at diagnosis $(\mathrm{P}=0.037)$. In addition to strong indication of lymphovascular invasion $(\mathrm{P}=0.000)$ and regional lymph node metastasis at diagnosis $(\mathrm{P}=0.025)$, high expression of CXCR7 was indicative of deeper dermis invasion $(\mathrm{P}=0.010)$. We found no significant association of CXCR4 and CXCR7 expression with other clinicopathological features including age, time delayed in diagnosis, longest diameter of lesion and surgical margin status.

Table 1. Clinicopathological characteristics in relation to CXCR4 and CXCR7 expression status

\begin{tabular}{|c|c|c|c|c|c|c|c|}
\hline \multirow[t]{2}{*}{ Variable } & \multirow{2}{*}{ Entire group $(\mathrm{n}=92)$} & \multicolumn{2}{|c|}{ CXCR4 } & \multirow{2}{*}{ Pvalue } & \multicolumn{2}{|c|}{ CXCR7 } & \multirow[t]{2}{*}{ P value } \\
\hline & & Low & High & & Low & High & \\
\hline Age, years & $68(35 \sim 87)$ & & & 0.799 & & & 0.983 \\
\hline$<70$ & $48(52.2)$ & 36 & 12 & & 37 & 11 & \\
\hline$\geq 70$ & $44(47.8)$ & 34 & 10 & & 34 & 10 & \\
\hline Delay in diagnosis, months & $36(2 \sim 244)$ & & & 0.575 & & & 0.071 \\
\hline$<24$ & $29(31.5)$ & 21 & 8 & & 19 & 10 & \\
\hline$\geq 24$ & $63(68.5)$ & 49 & 14 & & 52 & 11 & \\
\hline Longest diameter of lesion, $\mathrm{cm}$ & $5(1 \sim 12)$ & & & 0.815 & & & 0.634 \\
\hline$<5$ & $44(47.8)$ & 33 & 11 & & 33 & 11 & \\
\hline$\geq 5$ & $48(52.2)$ & 37 & 11 & & 38 & 10 & \\
\hline Surgical margin status & & & & 0.851 & & & 0.946 \\
\hline Positive & 18(19.6) & 14 & 4 & & 14 & 4 & \\
\hline Negative & 74(80.4) & 56 & 18 & & 57 & 17 & \\
\hline Depth of invasion & & & & 0.318 & & & 0.010 \\
\hline Intraepithelial & $45(48.9)$ & 36 & 9 & & 40 & 5 & \\
\hline Upper dermis & $21(22.8)$ & 17 & 4 & & 16 & 5 & \\
\hline Lower dermis & $26(28.3)$ & 17 & 9 & & 15 & 11 & \\
\hline Lymphovascular invasion & & & & 0.007 & & & 0.000 \\
\hline Yes & $16(17.4)$ & 8 & 8 & & 7 & 9 & \\
\hline No & $76(82.6)$ & 62 & 14 & & 64 & 12 & \\
\hline Regional lymph node metastasis & & & & 0.037 & & & 0.025 \\
\hline Yes & 19(20.7) & 11 & 8 & & 11 & 8 & \\
\hline No & $73(79.3)$ & 59 & 14 & & 60 & 13 & \\
\hline
\end{tabular}




\section{High expressions of CXCR4 and CXCR7 were associated with adverse prognosis}

In the survival analysis, high expression of CXCR4 was significantly associated with worse prognosis of EMPD in both PFS ( $\mathrm{P}<0.0001$, Fig. 2a) and CSS ( $\mathrm{P}=0.0013$, Fig. 2b). The same pattern of survival analysis was also observed with CXCR7 and PFS and CSS ( $\mathrm{P}<0.0001, \mathrm{P}<0.0001$, respectively; Fig. 2c, Fig. 2d). The combined prognostic model was established based on the expression of CXCR4 and CXCR7. Patients with concomitant high expression of both CXCR4 and CXCR7 showed the worst prognosis in PFS and CSS, and patients with both low expression of CXCR4 and CXCR7 showed better PFS and CSS $(\mathrm{P}<0.0001, \mathrm{P}<0.0001$, respectively; Fig. 2e, Fig. 2f).
Univariate and multivariate analyses were performed to assess the independent prognostic value of clinicopathological and immunohistochemical parameters for PFS and CSS. The results revealed that four parameters, including depth of invasion, lymphovascular invasion, regional lymph node metastasis at diagnosis, high expression of CXCR4 and CXCR7 and the combined prognostic model were associated with shorter PFS and CSS $(\mathrm{P}<0.05)$ in the univariate analysis (Table 2). Subsequent multivariate analysis suggested that depth of invasion, regional lymph node metastasis at diagnosis and the combined prognostic model were the independent prognostic factors of shorter PFS $(\mathrm{P}<0.05)$. Only depth of invasion and combined prognostic model impacted the CSS independently $(\mathrm{P}<0.05)$ (Table 3$)$.
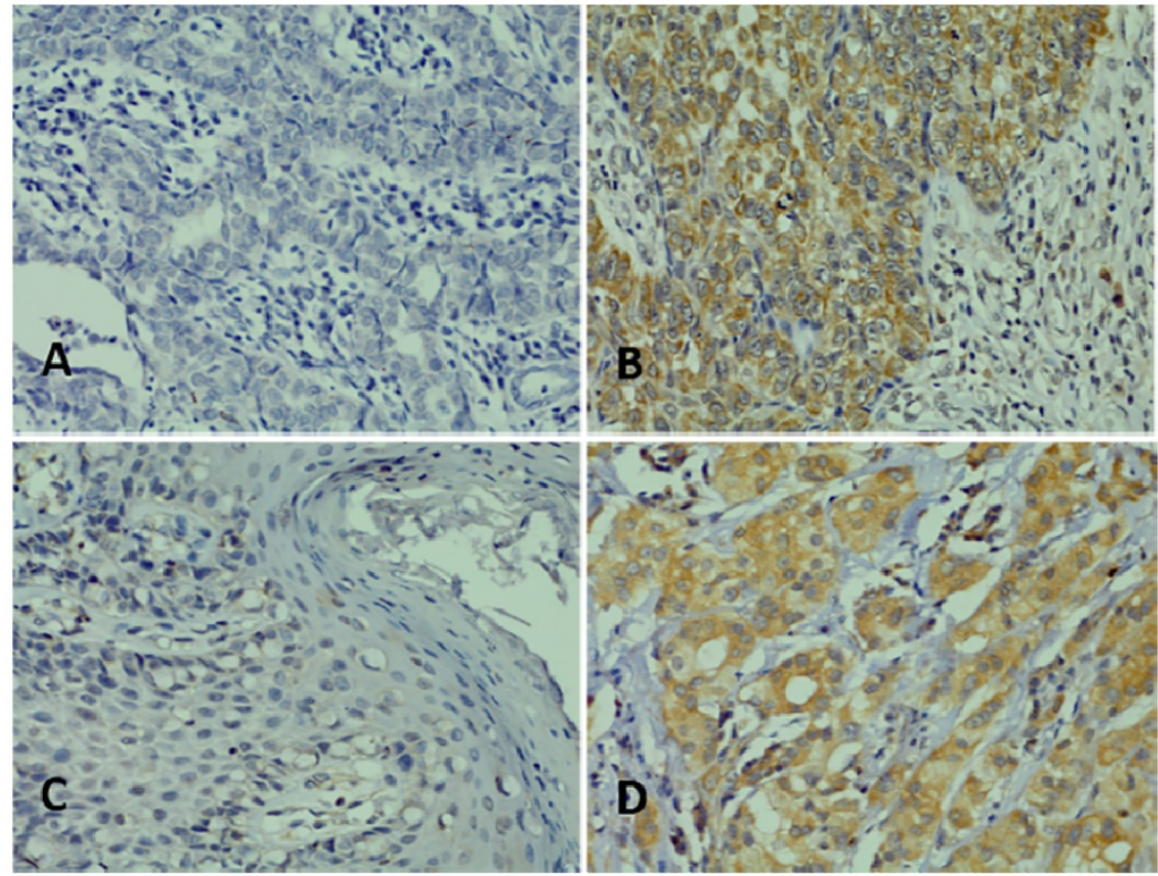

Figure 1. Representative images of immunohistochemical staining. (A) Low expression of CXCR4; (B) High expression of CXCR4; (C) Low expression of CXCR7; (D) High expression of CXCR7. Magnification, $\times 400$.

Table 2. Univariate analysis of clinicopathological parameters affecting disease-free survival and cancer-specific survival

\begin{tabular}{|c|c|c|c|c|}
\hline \multirow[t]{2}{*}{ Variables } & \multicolumn{2}{|l|}{ DFS } & \multicolumn{2}{|l|}{ CSS } \\
\hline & $\mathrm{HR}(95 \% \mathrm{CI})$ & P value & $\mathrm{HR}(95 \% \mathrm{CI})$ & P value \\
\hline Age ( $<70$ vs $\geq 70$ years) & $0.711(0.304 \sim 1.664)$ & 0.431 & $0.723(0.250 \sim 2.091)$ & 0.549 \\
\hline Delay in diagnosis ( $<24$ vs $\geq 24$ months) & $1.047(0.426 \sim 2.573)$ & 0.920 & $1.732(0.482 \sim 6.221)$ & 0.400 \\
\hline Longest diameter of lesion ( $<5 \mathrm{vs} \geq 5 \mathrm{~cm}$ ) & $0.691(0.298 \sim 1.600)$ & 0.388 & $0.573(0.198 \sim 1.658)$ & 0.304 \\
\hline Surgical margin status (positive vs negative) & $1.377(0.538 \sim 3.532)$ & 0.505 & $2.010(0.673 \sim 6.010)$ & 0.211 \\
\hline Depth of invasion (IE vs UD vs LD) & $4.705(2.465 \sim 8.982)$ & 0.000 & $13.209(3.460 \sim 50.435)$ & 0.000 \\
\hline Lymphovascular invasion (Yes vs No) & $20.040(7.353 \sim 54.618)$ & 0.000 & $51.210(10.767 \sim 243.564)$ & 0.000 \\
\hline Regional lymph node metastasis at diagnosis (Yes vs No) & $22.132(8.158 \sim 60.042)$ & 0.000 & $13.841(4.471 \sim 42.847)$ & 0.000 \\
\hline CXCR4 (High vs Low) & $5.549(2.320 \sim 13.275)$ & 0.000 & $5.098(1.706 \sim 15.235)$ & 0.004 \\
\hline CXCR7 (High vs Low) & $5.637(2.409 \sim 13.188)$ & 0.000 & $6.218(2.152 \sim 17.963)$ & 0.001 \\
\hline combined prognostic model (both high vs either high vs both low) & $4.389(2.472 \sim 7.795)$ & 0.000 & $4.445(2.171 \sim 9.100)$ & 0.000 \\
\hline
\end{tabular}

IE: Intraepithelial; UD: Upper dermis; LD: Lower dermis; both high: CXCR4 and CXCR7 high; either high: CXCR4 or CXCR7 high; both low: CXCR4 and CXCR7 low 

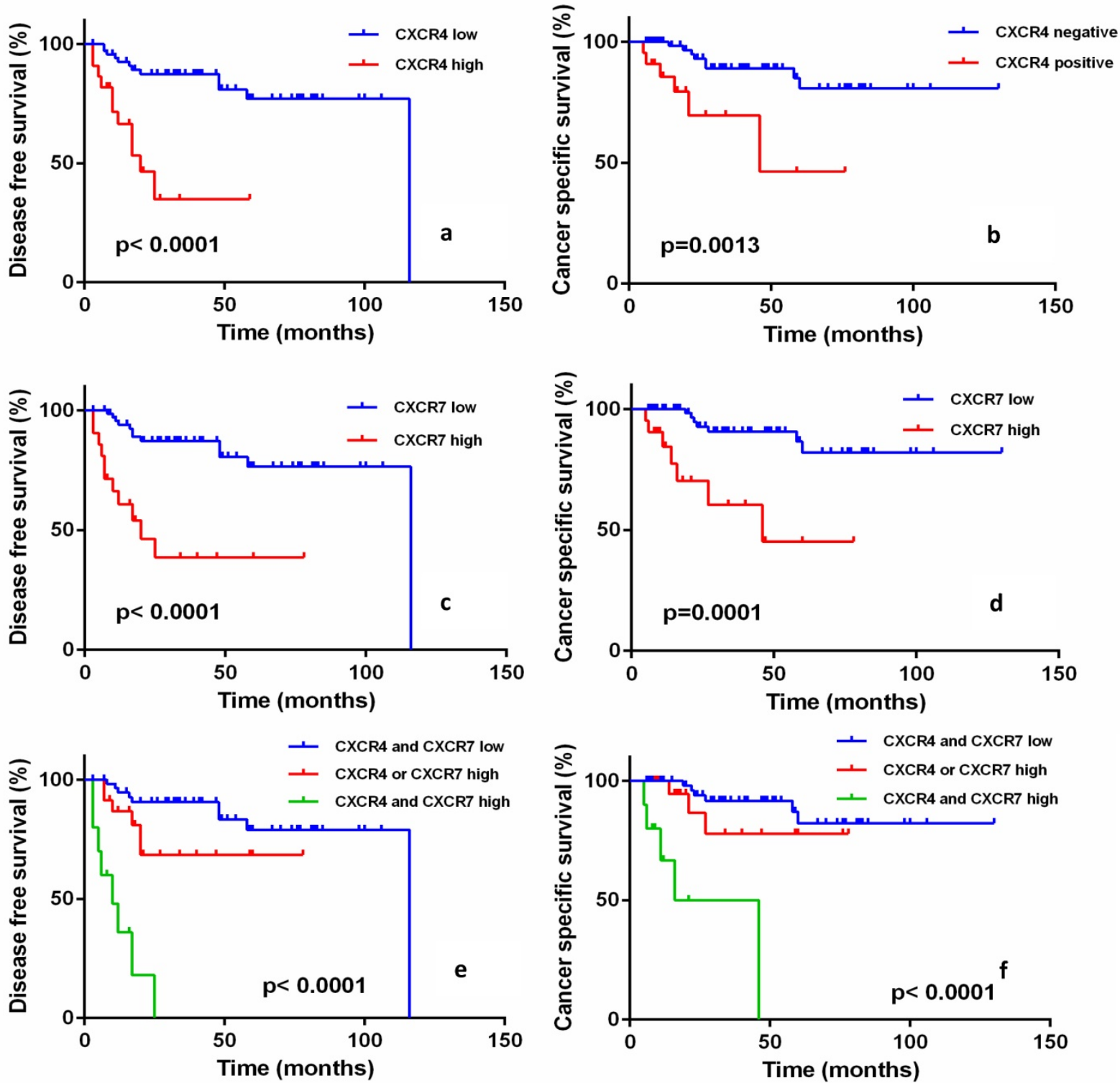

Figure 2. Progression-free survival (PFS) and cancer-specific survival (CSS) of EMPD patients according to the expression of CXCR4 and CXCR7. CXCR4 high expression patients versus CXCR4 low expression patients for PFS (a) and CSS (b) (log-rank $P<0.0001$ and $P=0.0013$, respectively). CXCR7 high expression patients versus CXCR7 low expression patients for PFS (c) and CSS (d) (log-rank $P<0.0001$ and $P=0.0001$ ). There was a significant difference among groups stratified according to CXCR4 and CXCR7 expression in PFS (e) and CSS (f) (log-rank $P<0.0001$ and $P<0.0001$ ), respectively. Patients with both high CXCR4 and CXCR7 expression had the worst prognosis.

Table 3. Multivariate analysis of clinicopathological parameters affecting disease-free survival and cancer-specific survival

\begin{tabular}{lllll}
\hline Variables & DFS & & CSS \\
\cline { 2 - 5 } & HR(95\%CI) & P value & HR(95\%CI) & P value \\
\hline Depth of invasion (IE vs UD vs LD) & $3.874(1.595 \sim 9.412)$ & 0.003 & $8.226(1.910 \sim 35.420)$ & 0.005 \\
Lymphovascular invasion(Yes vs No) & $0.128(0.016 \sim 1.062)$ & 0.057 & $0.843(0.062 \sim 11.394)$ & 0.898 \\
Regional lymph node metastasis at diagnosis(Yes vs No) & $34.166(5.909 \sim 197.544)$ & 0.000 & $7.748(0.972 \sim 61.782)$ & 0.053 \\
Combined prognostic model (both high vs either high vs both low) & $3.203(1.611 \sim 6.367)$ & 0.001 & $2.662(1.097 \sim 6.462)$ & 0.030 \\
\hline IE: Intraepithelial; UD: Upper dermis; LD: Lower dermis; both high: CXCR4 and CXCR7 high; either high: CXCR4 or CXCR7 high; both low: CXCR4 and CXCR7 low
\end{tabular}

\section{Discussion}

In the present study, we evaluated the expression of two chemokine receptors, CXCR4 and CXCR7, in a series of EMPD patients and correlated their expression patterns with clinicopathological characteristics and patient outcomes. We found that either high expression of CXCR4 or CXCR7 was indicative of lymphovascular invasion, regional lymph node metastasis at diagnosis and an adverse 
prognosis. In addition, high expression of CXCR7 was also correlated with the depth of invasion. Impressively, a combined prognostic model based on the expression of CXCR4 and CXCR7 could stratify the whole group into three categories, and showed that patients overexpressing both CXCR4 and CXCR7 experienced the worst prognosis. Our findings suggest that CXCR4 and CXCR7 could be highly relevant molecular markers of malignant potential and therapeutic targets for patients with EMPD.

Our findings showing that patients with high CXCR4 and CXCR7 expression exhibited the worst prognosis were consistent with similar findings in other tumors. In Florian's study, the expression status of CXCR4 and CXCR7 was examined in 249 pancreatic adenocarcinoma patients and 47 patients that were CXCR7-positive also showed CXCR4 expression, which underlines the functional affiliation between these two molecules. ${ }^{[21]}$ In Heinrich's study, immunohistochemical staining of 51 human pancreatic cancer specimens demonstrated high frequency of CXCR4 and CXCR7 co-expression: 37 showed double staining and 5 showed single staining, while 9 had no staining., ${ }^{[19]} \mathrm{D}^{\prime}$ Alterio et al. demonstrated that concomitant high expression of CXCR4 and CXCR7 ( $\mathrm{P}=0.0235)$ was an independent prognostic factor for renal cell carcinoma. $\left.{ }^{22}\right]$

Under pathological conditions, CXCR4 and CXCR7 signaling mediates several cellular effects affecting leukocyte recruitment, neovascularization and tumor progression. Activation of MAP and Akt kinase pathways promotes cell survival and proliferation, and transcriptional regulation of multiple genes affects angiogenesis, invasion and adhesion of cells. When co-expressed, CXCR4 and CXCR7 may form homo- and heterodimers, and heterodimerization seems to play an important role in the modulation of downstream signaling. ${ }^{[23-25]}$ CXCR4 and CXCR7 co-expression on the same cells resulted in stronger calcium flux and more robust phosphorylation of MAPKp42/44 in response to SDF-1 stimulation compared with cells that express only CXCR4.[26] This suggests that the heterodimeric receptor potentially might activate a broader panel of intracellular pathways than activation of only one receptor. ${ }^{[27,28]}$ This may explain why the patients high expressing both receptors showed worse prognosis compared with the other groups.

For EMPD patients, identification of invasive disease is of great value in clinical practice. Consistent with previous published studies, our study also suggested that depth of invasion was an important prognosticator in EMPD. Patients diagnosed as invasive disease tend to experience rapid progression and adverse prognosis. ${ }^{[29-33]}$ However, it is difficult to distinguish invasive disease with noninvasive disease before surgery. Thus, the identification of reliable biomarkers to distinguish invasive and noninvasive disease at the time of biopsy is desperately needed. A retrospective study including 44 specimens from 38 primary EMPD cases found that invasive lesions and metastatic lymph nodes tended to express significantly higher MUC5AC levels than in situ lesions $(\mathrm{P}<0.01) \cdot{ }^{[34]}$ Other studies reported that Stat5a, E-cadherin and even FDG PET/CT may play some role in the invasion of EMPD. ${ }^{[35,36]}$ Aoyagi et al. reported that combined high expression of $\mathrm{Ki}-67$ and cyclin D1 were useful for the early detection of micro-invasive EMPD. [3] However, several reasons have prevented the use of these biomarkers. First, due to the rarity of EMPD, most of these cohorts consisted of relatively small sample sizes, especially patients with invasive disease. Furthermore, the short follow-up also restricted the validation and application of these markers. In our study, we evaluated the expression of CXCR7 in 92 specimens, among which 47 were invasive cases. Over the median follow-up of 34 months (range 6-130 months), one quarter of the patients experienced clinical recurrence and over 15\% patients died of EMPD. We found that high expression of CXCR7 closely correlated with invasive disease. This suggests that patients with high expression of CXCR7 at preoperative biopsy may be linked with invasive disease. Aggressive treatment might be advisable for these patients, including wider and deeper excision of primary lesion with or without adjuvant radiation therapy, and intensive follow-up. To decrease the possibility of local recurrence and distant metastasis, complementary resection may also be recommended.

Although the clinical significance of CXCR4 and CXCR7 in EMPD has been revealed, several limitations of this study warrant further discussion. First, this study was conducted in a single center. An independent external cohort is necessary to confirm our findings. Second, our results were based on a retrospective analysis, which may have resulted in selection bias because of the various therapeutic strategies that were performed. Therefore, prospective studies are also needed to validate our findings. Finally, functional studies should be conducted to uncover the biological mechanisms of CXCR4 and CXCR7 in EMPD.

\section{Acknowledgements}

This study was supported in part by a grant from the Natural Science Foundation of Shanghai Municipality (No. 16ZR1406500), a grant from the Outstanding Young Talent Training Plan of Shanghai Municipal Commission of Health and Family 
Planning (No. XYQ2013102), and a grant from Shanghai Cancer Research Charity Center.

\section{Author Contributions}

KC and GXL designed the study, analyzed and interpreted the clinical data, and wrote the manuscript. $\mathrm{KC}$ and $\mathrm{ZWJ}$ conducted the experiments. YYQ and YW helped with patient follow-up. YYK, BD and DWY supervised the project and revised the manuscript. All authors approved the final version and agreed to publish the manuscript.

\section{Competing Interests}

The authors have declared that no competing interest exists.

\section{References}

[1] Lloyd J, Evans DJ, Flanagan AM. Extension of extramammary Paget disease of the vulva to the cervix. J Clin Pathol. 1999; 52: 538-540.

[2] Ohara K, Fujisawa Y, Yoshino K, et al. A proposal for a TNM staging system for extramammary Paget disease: Retrospective analysis of 301 patients with invasive primary tumors. J Dermatol Sci. 2016; 83: 234-239.

[3] Aoyagi S, Akiyama M, Shimizu H, et al. High expression of Ki-67 and cyclin D1 in invasive extramammary Paget's disease. J Dermatol Sci. 2008; 50: 177-184.

[4] Tanaka R, Sasajima Y, Tsuda H, et al. Human epidermal growth factor receptor 2 protein overexpression and gene amplification in extramammary Paget disease. Br J Dermatol. 2013; 168: 1259-1266.

[5] Shiomi T, Noguchi T, Nakayama H, et al. Clinicopathological study of invasive extramammary Paget's disease: Subgroup comparison according to invasion depth. J Eur Acad Dermatol Venereol. 2013; 27: 589-592.

[6] Hatta N, Yamada M, Hirano T, et al. Extramammary Paget's disease: Treatment, prognostic factors and outcome in 76 patients. Br J Dermatol. 2008; 158: 313-318.

[7] Dai B, Kong YY, Chang K, et al. Primary invasive carcinoma associated with penoscrotal extramammary Paget's disease: A clinicopathological analysis of 56 cases. Bju Int. 2015; 115: 153-160.

[8] Craig MJ, Loberg RD. CCL2 (Monocyte Chemoattractant Protein-1) in cancer bone metastases. Can Met Rev. 2006; 25: 611-619.

[9] Homey B, Muller A, Zlotnik A, et al. Chemokines: Agents for the immunotherapy of cancer?. Nat Rev Immunol. 2002; 2: 175-184.

[10] Zlotnik A. Chemokines in neoplastic progression. Semin Can Biol. 2004; 14: 181-185.

[11] Strieter RM, Belperio JA, Phillips RJ, et al. CXC chemokines in angiogenesis of cancer. Semin Can Biol. 2004; 14: 195-200.

[12] Balabanian K, Lagane B, Infantino S, et al. The chemokine SDF-1/CXCL12 binds to and signals through the orphan receptor RDC1 in T lymphocytes. J Biol Chem. 2005; 280: 35760-35766.

[13] Miao Z, Luker KE, Summers BC, et al. CXCR7 (RDC1) promotes breast and lung tumor growth in vivo and is expressed on tumor-associated vasculature. Proc Natl Acad Sci USA. 2007; 104: 15735-15740.

[14] Wang J, Shiozawa Y, Wang J, et al. The role of CXCR7/RDC1 as a chemokine receptor for CXCL12/SDF-1 in prostate cancer. J Biol Chem. 2008; 283: 4283-4294.

[15] Tarnowski M, Grymula K, Reca R, et al. Regulation of expression of stromal-derived factor-1 receptors: CXCR4 and CXCR7 in human rhabdomyosarcomas. Mol Can Res. 2010; 8: 1-14.

[16] Hattermann K, Held-Feindt J, Lucius R, et al. The chemokine receptor CXCR7 is highly expressed in human glioma cells and mediates antiapoptotic effects. Can Res. 2010; 70: 3299-3308.

[17] Edge SB, Compton CC. The American Joint Committee on Cancer: The 7th edition of the AJCC cancer staging manual and the future of TNM. Ann Surg Oncol. 2010; 17: 1471-1474

[18] Kononen J, Bubendorf L, Kallioniemi A, et al. Tissue microarrays for high-throughput molecular profiling of tumor specimens. Nat Med. 1998; 4: 844-847.

[19] Heinrich EL, Lee W, Lu J, et al. Chemokine CXCL12 activates dual CXCR4 and CXCR7-mediated signaling pathways in pancreatic cancer cells. J Transl Med. 2012; 10: 68.

[20] Guo JC, Li J, Zhou L, et al. CXCL12-CXCR7 axis contributes to the invasive phenotype of pancreatic cancer. Oncotarget. 2016; 7: 62006-62018.

[21] Gebauer F, Tachezy M, Effenberger K, et al. Prognostic impact of CXCR4 and CXCR7 expression in pancreatic adenocarcinoma. J Surg Oncol. 2011; 104: $140-145$.
[22] D'Alterio C, Consales C, Polimeno M, et al. Concomitant CXCR4 and CXCR7 expression predicts poor prognosis in renal cancer. Curr Cancer Drug Targets. 2010; 10: 772-781.

[23] Decaillot FM, Kazmi MA, Lin Y, et al. CXCR7/CXCR4 heterodimer constitutively recruits beta-arrestin to enhance cell migration. J Biol Chem. 2011; 286: 32188-32197.

[24] Levoye A, Balabanian K, Baleux F, et al. CXCR7 heterodimerizes with CXCR4 and regulates CXCL12-mediated G protein signaling. Blood. 2009; 113: 6085-6093.

[25] Luker KE, Steele JM, Mihalko LA, et al. Constitutive and chemokine-dependent internalization and recycling of CXCR7 in breast cancer cells to degrade chemokine ligands. Oncogene. 2010; 29: 4599-4610.

[26] Sierro F, Biben C, Martinez-Munoz L, et al. Disrupted cardiac development but normal hematopoiesis in mice deficient in the second CXCL12/SDF-1 receptor, CXCR7. Proc Natl Acad Sci USA. 2007; 104: 14759-14764.

[27] Mellado M, Rodriguez-Frade JM, Vila-Coro AJ, et al. Chemokine receptor homo- or heterodimerization activates distinct signaling pathways. Embo J. 2001; 20: 2497-2507.

[28] Maksym RB, Tarnowski M, Grymula K, et al. The role of stromal-derived factor-1--CXCR7 axis in development and cancer. Eur J Pharmacol. 2009; 625: $31-40$.

[29] Lam C, Funaro D. Extramammary Paget's disease: Summary of current knowledge. Dermatol Clin. 2010; 28: 807-826.

[30] Ito Y, Igawa S, Ohishi $Y$, et al. Prognostic indicators in 35 patients with extramammary Paget's disease. Dermatol Surg. 2012; 38: 1938-1944.

[31] Hatta N, Yamada M, Hirano T, et al. Extramammary Paget's disease: Treatment, prognostic factors and outcome in 76 patients. Br J Dermatol. 2008; 158: 313-318.

[32] Karam A, Dorigo O. Treatment outcomes in a large cohort of patients with invasive Extramammary Paget's disease. Gynecol Oncol. 2012; 125: 346-351.

[33] Crawford D, Nimmo M, Clement PB, et al. Prognostic factors in Paget's disease of the vulva: A study of 21 cases. Int J Gynecol Pathol. 1999; 18: 351-359.

[34] Hata H, Abe R, Hoshina D, et al. MUC5AC expression correlates with invasiveness and progression of extramammary Paget's disease. J Eur Acad Dermatol Venereol. 2014; 28: 727-732.

[35] Liu H, Urabe K, Uchi H, et al. Expression and prognostic significance of Stat5a and E-cadherin in extramammary Paget's disease. J Cutan Pathol. 2007; 34: 33-38.

[36] Li ZG, Qin XJ. Extensive invasive extramammary Paget disease evaluated by F-18 FDG PET/CT: A case report. Medicine (Baltimore). 2015; 94: e371. 